\title{
MODELLING IN MODELICA OF A PNEUMATIC MUSCLE: APPLICATION TO MODEL AN EXPERIMENTAL SET-UP
}

\author{
Aron Pujana-Arrese \\ Javier Arenas \\ Iban Retolaza \\ Ana Martinez-Esnaola \\ Joseba Landaluze \\ IKERLAN Technological Research Centre \\ Arizmendiarrieta, 2. E20500 Arrasate. Spain \\ E-mail: APujana@ikerlan.es
}

\section{KEYWORDS \\ Modelica, pneumatic muscle, model, robotic arm.}

\begin{abstract}
The characteristics of pneumatic artificial muscles - or McKibben muscles - make them very interesting for the development of robotic applications that feature low impedance in terms of their interaction with the environment, such as can be the case with orthoses or certain wearable robots. In order to research the applicability of these actuators in industrial applications, an experimental one-degree-of-freedom set-up based on pneumatic muscles manufactured by Festo has been built at Ikerlan. This paper presents the modelling of a pneumatic muscle in Modelica as a new component. After that, the paper describes the set-up constructed and shows the complete model in Dymola/Modelica, in addition to validation of the model with some experimental data.
\end{abstract}

\section{INTRODUCTION}

Most robots use actuators and control systems which feature high mechanical impedance, both for historical and technological reasons. In contrast, there is evidence in the natural world that natural impedances in animals are quite low. Nowadays there are a great many robotic applications, as for instance protheses, orthoses and wearable robots, where low impedance is required in terms of interaction with the environment. The observation of nature and the use of biologicallyinspired components (sensors and actuators) open up new ways in the design of biomimetic robotic devices. Electroactive polymers (EAPs) are one of the new technologies existing, although their applicability remains far from industrial applications. Pneumatic artificial muscles - or McKibben muscles - are a very interesting alternative.

Unfortunately, pneumatic artificial muscles evidence certain very non-linear force-length characteristics, like animal muscles, and controlling them and obtaining the performance features demanded by some applications are no easy issues. There has been quite a lot of activity regarding the modelling and control in recent years (Tondu and Lopez 2000; Colbrunn et al. 2001; Petrovic 2002; Schröder et al. 2003).

For the purpose of researching the applicability of pneumatic muscles in industrial applications and in the development of orthoses and wearable robots, an experimental one-DoF set-up based on pneumatic muscles manufactured by Festo has been designed and constructed at the Ikerlan research centre.

This paper first and foremost presents a brief review of the pneumatic muscle models that feature in literature. The analytical model chosen is then described, followed by the modelling of a new component in Dymola/Modelica as an extension of the PneuLib library. Following the description of the experimental set-up that has been constructed, its complete modelling is shown in the aforementioned package. Lastly, validation of the model with experimental data is shown.

\section{BRIEF REVISION OF PNEUMATIC MUSCLE'S MODELS}

Membrane or pneumatic muscle actuators are highly non-linear systems. Many authors have worked on the idea of obtaining a model that represents the behaviour of these devices. The aim of the model is to relate the pressure and length of the pneumatic muscle to the force it exerts along its entire axis. Figure 1 shows the wellknown outline of the constitution of the pneumatic muscle. $L$ is the length of the cylinder and $D$ the diameter. Assuming inextensibility of the mesh material, the geometric constants of the system are the thread length $b$ and the number of turns $n$ for a single thread. $\alpha$ is the angle between the thread and the long axis of the cylinder. The angle changes as the length of the muscle changes.

From these premises, Tondu and Lopez (2000) have carried out a mathematical development based on the application of theorem of virtual work, which has led them to obtain the following equation: 


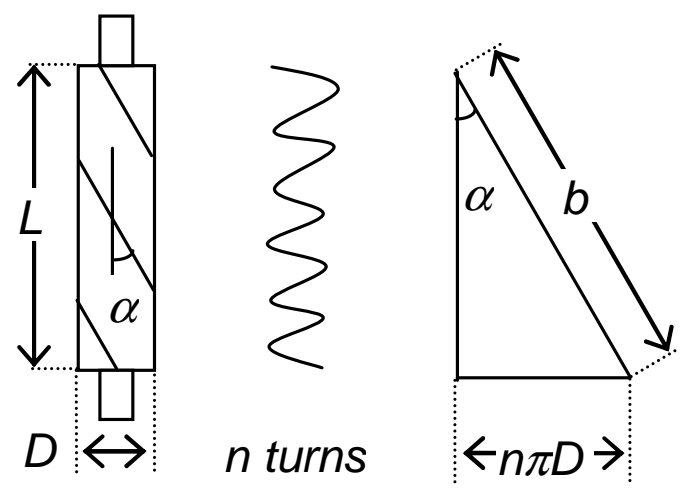

Figure 1: Geometric model of a pneumatic muscle

$$
F(\varepsilon, P)=\left(\pi r_{0}^{2}\right) P\left[a(1-\varepsilon)^{2}-b\right]
$$

where:

$$
\begin{aligned}
& \mathcal{E}=\frac{\left(l_{0}-l\right)}{l_{0}} \quad 0 \leq \varepsilon \leq \varepsilon_{\max } \\
& a=\frac{3}{\tan ^{2}\left(\alpha_{0}\right)}, \quad b=\frac{1}{\sin ^{2}\left(\alpha_{0}\right)}
\end{aligned}
$$

$r_{0}$ being the nominal inner radius, $l$ the length of the muscle, $l_{0}$ the initial nominal length, $P$ the pressure and $\alpha_{0}$ the initial angle between the membrane fibres and the muscle axis.

In this initial approximation, it has been assumed that the thickness of the muscle walls is very small and it may also be assumed that pressure is transmitted evenly throughout the membrane. However, there is a major phenomenon which Tondu and Lopez (2000) consider to improve equation (1): the fact that the shape of the muscle is not cylindrical on the ends, but rather is flattened. To represent this, they incorporate a correction factor $k$, been equation (1) as follows:

$$
F(\varepsilon, P)=\left(\pi r_{0}^{2}\right) P\left[a(1-k \varepsilon)^{2}-b\right]
$$

Tondu and Lopez (2000) establish two options for the choice of parameter $k$ : On the one hand, they propose considering a constant value for $k$ which may vary depending on the material that the muscle is made of and, on the other, they suggest making the parameter $k$ depend on the pressure in the muscle at any given time. According to their studies, this last-mentioned option is the one that provides the most complete model.

Other analytical expressions for calculating the force of a pneumatic muscle may be found in technical literature. Thus, in (Colbrunn et al. 2001), (Petrovic 2002) and (Umetani et al. 1999), formulas equivalent to (2) are put forward based on the same principles as Tondu and Lopez (2002), and may be readily deduced from each other. In all of them is reflected the fact that the force exerted by the muscle has a linear relation with the pressure inside it and non-linear with the contraction. However, calculating the parameters in an analytical way is complicated and inaccurate, which is why they need to be adjusted via experimental trials.

\section{PNEUMATIC MUSCLE MODEL IN MODELICA}

\section{Model selection}

As has been explained in the previous section, there is a relation between the force exerted by the muscle along its axis and the pressure and contraction it experiences. For the model put forward in this work, the equation (3) proposed in (Petrovic 2002) has initially been chosen due to the simplicity of $2^{\text {nd }}$ order polynomial adjustment.

$$
F=\left(D_{1}+D_{2} \cdot q+D_{3} \cdot q^{2}\right) P
$$

with $q$ being the displacement $q=l_{0}-l$.

However, in the experimental trials carried out and which are shown later, it was noted that to identify the coefficients of equation (3), it had to incorporate a new term, independent from the pressure and dependent on contraction. Therefore, the ratio between force, pressure and contraction in the muscles takes the following form:

$$
F=\left(D_{1}+D_{2} \cdot q+D_{3} \cdot q^{2}\right) P+\varphi(q)
$$

This correction term $\varphi(q)$ introduced into the model will be determined in each case from the experiences needed to identify the parameters of the equation (4) taken into consideration. In conclusion, the expression that reflects the equation (4) is that which is implemented in Modelica in order to govern the force exerted by the muscle at all times. The parameters of the model may be identified analytically and approximately according to the models that appear in (Umetani et al. 1999; Colbrunn et al. 2001; Petrovic 2002), or experimentally in each case, as is also recommended in the aforementioned references.

\section{Model implementation in Modelica}

The model analyzed and selected in the previous section has been implemented by using the good qualities of the modelling language of physical systems known as Modelica. It is suited for multi-domain modelling, for example and in this case, mechatronic models in robotics, involving pneumatic, mechanical and control subsystems.

The muscle model has been designed, ensuring its connectivity with other objects described in Modelica, in order to construct different mechanical-pneumatic systems. The model consists of two mechanical connectors that correspond to the muscle anchorage 
parameter Real l_nom=200 "muscle nominal lenght (mm)"; parameter Real alfa=25 "angle of the fibres of the muscle in degrees"; parameter Real $\mathbf{d} \mathbf{0}=20$ "nominal diameter of the muscle"; parameter Modelica.SIunits.CoefficientOfHeatTransfer h (finalmin=0)=200.0 "coefficient of heat transfer";

parameter Modelica.SIunits.Area surface_heat_transfer $=0.0126$ "area for heat transfer";

parameter Modelica.SIunits.Pressure $\mathbf{p} \_$initial= environment.p_atmosphere "initial pressure in muscle" annotation (Dialog(tab="Initial", group="Pressure"));

parameter Modelica.SIunits.Pressure pWarning= environment.pWarning "warning is issued when pressure below this value" annotation (Dialog(tab="Warning"));

parameter String StringWarning = environment.StringWarning "text is issued when pressure below this value" annotation (Dialog(tab="Warning"));

Modelica.SIunits.Temp_K

temp_in_out(nominal=environment.temp_surroundings) "temperature of entering or leaving air";

Modelica.SIunits.Pressure p_muscle(nominal=5e5, stateSelect=StateSelect.avoid) "muscle pressure";

Modelica.SIunits.Temp_K temp_muscle(stateSelect=StateSelect.always,start= environment.temp_surroundings,nominal= environment.temp_surroundings) "muscle temperature";

Modelica.SIunits.Velocity v_rel(stateSelect=StateSelect.prefer) "relative velocity between between flange_a and flange_b";

Modelica.SIunits.Volume vol_eff(stateSelect=StateSelect.avoid) "effective volume as a function of stroke";

Modelica.SIunits.Mass m "gas mass in muscle";

Real force "muscle mechanic force";

Real phi;

Real d "muscle diameter";

Real vol_eff_cil "muscle volume";

Real vol_achat "volume of the flattened zone";

Real h_achat "height of the flattened zone";

outer PneuLib.Environment environment;

parameter Real alfa0=alfa*Modelica.Constants.pi/180 "angle of the fibres of the muscle (radians)";

parameter Real $\mathbf{b}=$ l_nom/cos(alfa0) "lenght of the fibres";

parameter Real $\mathbf{n}=((1 /(\cos ($ alfa 0$)) \wedge 2)-1) * 1 \_n o m \wedge 2 /$

(d0*Modelica.Constants.pi) "number of turns of each fibre";

Figure 2: Definition of parameters and local variables

points, and a pneumatic port that makes it possible to connect to an external pneumatic circuit. Moreover, an output has been included so as to establish the force exerted at all times by this pneumatic actuator.

Three main parameters have been taken into consideration that characterize the physical properties of this type of muscle: nominal length $l \_n o m$, nominal diameter $d \_0$ and the angle between the fibres and the muscle axis alfa. Other magnitudes that may be considered as parameters are, for instance, the heat transfer coefficient surface_heat_transfer or the maximum pressure beyond which the system delivers a warning message p_warning. The declaration in Modelica language of all these parameters is shown in Figure 2, in addition to the local variables used in the class.
[1] phi= if $((1-($ s_rel*1000/1_nom $))>0.008)$ then $0.00045804 *\left(1 \_n o m-s \_r e l * 1000\right) \wedge 4$ $-0.053424 *\left(1 \_\right.$nom-s rel $\left.* 1000\right) \wedge 3$

$+2.0189 *\left(1 \_\right.$nom-s_rel $\left.* 1000\right) \wedge 2$

$-43.691 *\left(1 \_\right.$nom-s_rel*1000)+61.62 else -9.2101;

[2] force $=\left(280-2.2 *\left(1 \_n o m-S \_r e l * 1000\right)+0.033 *\left(1 \_n o m-\right.\right.$ s_rel*1000)^2)*port_1.p/1e5+phi;

[3] flange_b.f $=$ if (force $<0.0)$ then 0.0 else force;

[4] y= flange_b.f "output port";

[5] v_rel = der(s_rel);

[6] d= (b^2 - (s_rel*1000)^2)/(Modelica.Constants.pi*n);

[7] vol_eff_cil= $(1 / 4 *$ Modelica.Constants.pi*d^2*s_rel*1000)/1e9 "volume without flattening";

[8] h_achat= if noEvent $(d>d 0)$ then $\operatorname{sqrt}(d \wedge 2 / 4-d 0 \wedge 2 / 4)$ else 0.0001 ;

[9] vol_achat $=($ Modelica.Constants.pi*h_achat $/ 6) *(3 * d \wedge 2 / 4+$ $3 * \mathrm{~d} \wedge 2 / 4+$ h_achat $\wedge 2) / 1 \mathrm{e} 9$;

[10] vol_eff= vol_eff_cil + $2 *$ vol_achat "volume taking into account the flattening";

[11] temp_in_out $=$ if (port_1.m_dot $>0$ ) then environment.temp_surroundings else temp_muscle;

[12] p_muscle = port_1.p;

[13] der(m) = port_1.m_dot;

[14] p_muscle*vol_eff $=\mathrm{m} *$ environment.R*temp_muscle "ideal gas equation";

[15] m*der(temp_muscle) + temp_muscle*port_1.m_dot = environment.gamma* temp_in_out*port_1.m_dot + der(vol_eff)*port_1.p/environment.c_v -

h*surface_heat_transfer*(temp_muscle environment.temp_surroundings)/environment.c_v "continuity equation";

Figure 3: Equations of the model in Modelica

The equations that characterize the physical behaviour of the muscle, and which are grouped together within the equation section, are those shown in Figure 3. The first equations [1-5] are used to describe the mechanical force exerted by the muscle depending on its length s_rel and according to the mathematical model selected and reflected by equation (4). The displacement of the muscle is considered as a state variable of the model.

The volume vol_eff is meticulously calculated using the following set of equations [6-10], as even the flattening experienced on the ends of the muscle when it is contracted (vol_achat) is taking into consideration for calculation purposes.

The volume, together with the value of other state variables of the model such as the temperature temp_muscle on the inside and the air mass $m$, forms an essential part of thermo-dynamic equations of the muscle. The first of these [14] refers to the ideal gas equation, and the last is the thermo-dynamic continuity equation [15].

The following equations represent the same last two equations mentioned before but written in a more 


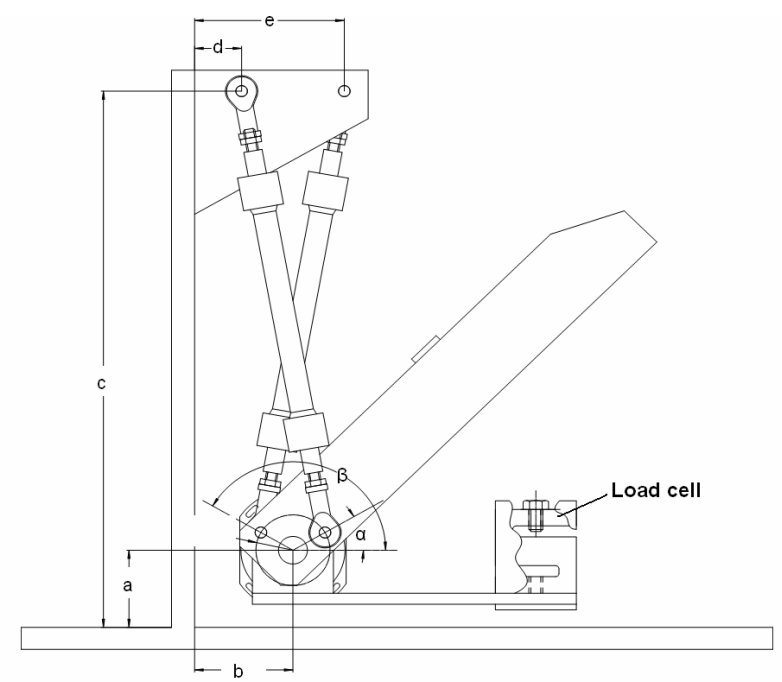

Figure 4: Geometric model of the robotic arm

understandable format. Equation (5) is the state equation of ideal gas:

$$
P \cdot V_{\text {eff }}=m \cdot R \cdot T_{\text {muscle }}
$$

where $m$ is the air mass and $T_{\text {muscle }}$ the temperature in the inside. $V_{\text {eff }}$ is the effective volume of the muscle.

The last of the equations taking into account corresponds to the thermodynamic continuity equation:

$$
\begin{aligned}
& m \cdot \frac{d T_{\text {muscle }}}{d t}+T_{\text {muscle }} \cdot m_{\text {dot }}= \\
& \gamma \cdot T_{\text {inout }} \cdot m_{\text {dot }}+\frac{d V_{\text {eff }}}{d t} \cdot P / C V-h \cdot s \cdot\left(T_{\text {muscle }}-T_{\text {sou }}\right) / C V
\end{aligned}
$$

With $m_{\text {dot }}$ being air mass flow rate, $\gamma$ the ratio of specific heat capacities, $T_{\text {inout }}$ the temperature of air entering or leaving muscle, $C v$ specific heat capacity at constant volume, $s$ the heat transfer surface area, $h$ heat transfer coefficient and $T_{\text {sou }}$ the temperature of the surroundings.

\section{DESCRIPTION OF EXPERIMENTAL SET-UP}

A human arm orthosis-type application has been taken into consideration when designing the set-up. To this end and albeit with a single degree of freedom, it was considered that it should allow for the greatest angular displacement possible, and that it should be able to transport the greatest mass possible at the tip (emulating a weight borne by the hand). On the other hand, however, it needed to be confined to the length of the pneumatic muscles. In seeking a compromise between all the specifications, a displacement of around $60^{\circ}$ and a maximum mass to be moved at the tip of $8 \mathrm{~kg}$ were set. By trying to minimize the length of the muscle required, the design focused on the mechanism that would enable the arm and inertias to rotate with good dynamics from the two precise muscles.

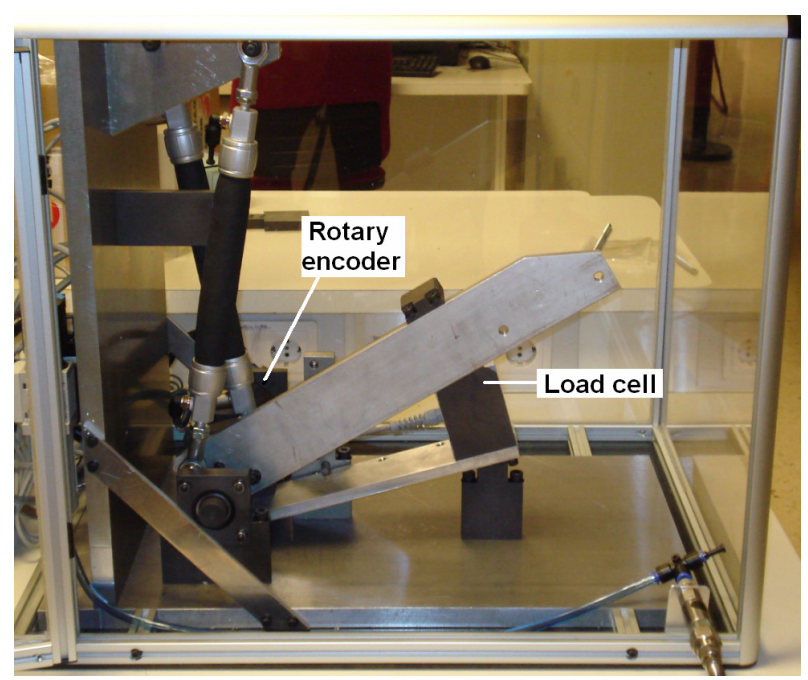

Figure 5: Picture of the experimental set-up

The pneumatic muscle that was chosen was the DMSP20-200N manufactured by Festo, and the resultant mechanism is that shown in Figure 4. The parameter values that define the mechanism are:

$$
\begin{aligned}
& a=5 \mathrm{~mm} ; b=85 \mathrm{~mm} ; c=491 \mathrm{~mm} ; d=40.6 \mathrm{~mm} ; \\
& e=129.4 \mathrm{~mm} ; \alpha=0^{\circ}-60^{\circ} ; \beta=120^{\circ}-180^{\circ}
\end{aligned}
$$

A distance $L(\mathrm{~mm})$ emerges from these values between the ends (joining points of the mechanism) of the pneumatic muscles of:

$$
L=\sqrt{175059+2841.6 \cdot \cos \alpha-26624 \cdot \sin \beta}
$$

When the muscles are without pressure, there is a distance of $423 \mathrm{~mm}$, with the length of the muscle fibre being $200 \mathrm{~mm}$. The centre of the arm mass with regard to the centre of rotation is at a height of $17.6 \mathrm{~mm}$ and at a horizontal distance of $205 \mathrm{~mm}$, considering that the arm is in the horizontal position. The arm mass is 0.987 $\mathrm{kg}$. The centre of the additional masses placed on the end of the arm (up to a maximum $8 \mathrm{~kg}$ ) would be at a height of $-24 \mathrm{~mm}$ and at a horizontal length of $367 \mathrm{~mm}$ with regard to the centre of rotation, always bearing in mind that the arm is in the horizontal position. Figure 5 shows an image of the model that has been constructed. The prototype may be rotated so that the arm moves in a horizontal plane and the effects of gravity are therefore cancelled out.

A Festo MPYE-5-1/8HF pneumatic servovalve is initially used for actuation, with working pressure being 6 bar. Festo SDE-D10 pressure sensors are used to establish the entry pressure of each muscle. The set-up includes a FAGOR S-D90 encoder which supplies 180000 pulses per turn, so as to measure the rotation angle of the arm accurately. A load cell is also included on the lower stop of the model (Figure 4 and Figure 5), so that the force exerted by the arm against this stop may be measured. The stop may be fastened at different 


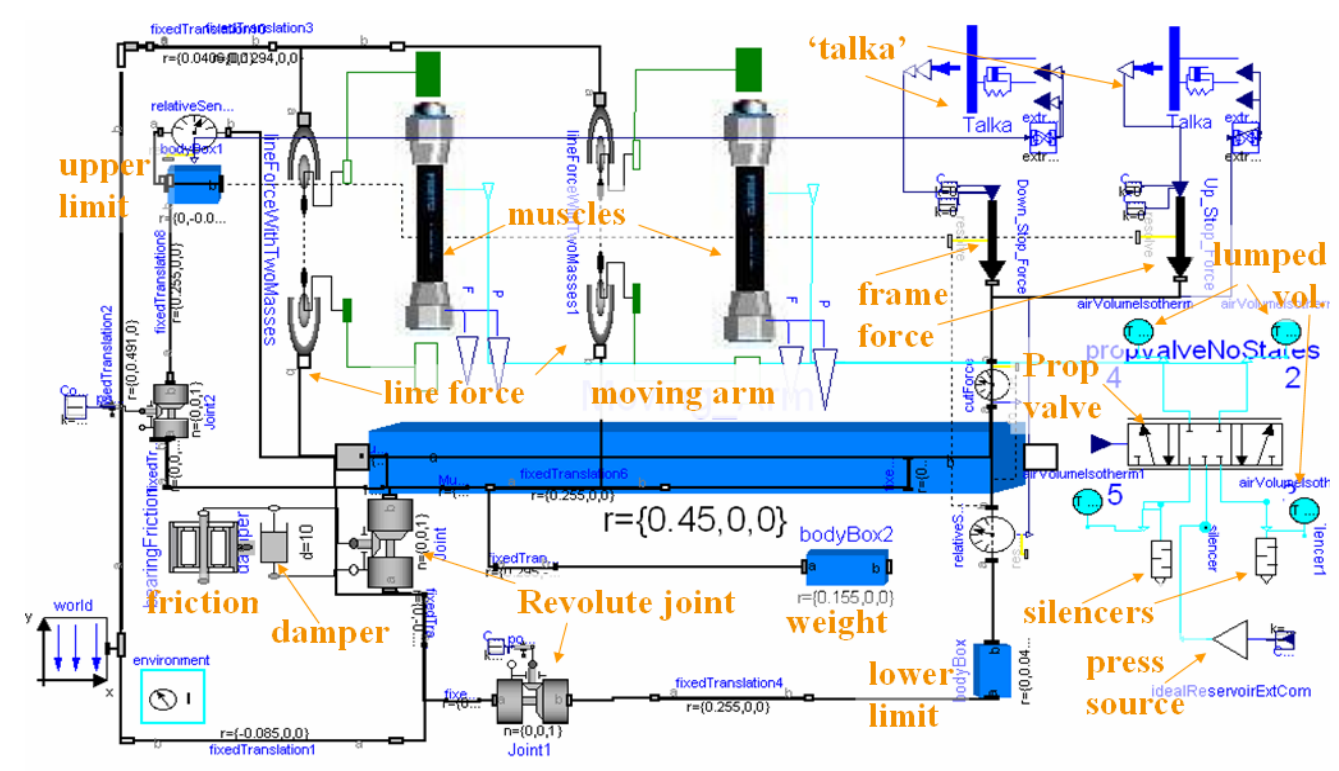

Figure 6: Model of the whole system in Modelica

angles, whereby the force exerted by the muscles may be calculated at different lengths and different pressures.

PIP8 hardware made by the MPL company is used as a controller, which is similar to the MathWorks xPCTargetBox and in which the cards needed to control the system have been incorporated. The control algorithms are implemented in Simulink and code generated and downloaded in the aforementioned hardware by means of the MathWorks RTW.

\section{MODELLING OF THE EXPERIMENTAL SET-UP}

For composition of the model, connection properties for linking objects provided by the graphic interface of Dymola have been used. Figure 6 shows the graphic representation of the whole model designed in Dymola. Its most significant components are explained in detail in the following paragraphs.

In constructing the model, elements which have been expressly developed for this application (such as the pneumatic muscle model), coexist with components belonging to commercial libraries. There exist components of different domains which are related to each other by special objects that carry out the connection work between the mechanical part and the pneumatic part.

The element that represents the metal arm is an object known as bodyBox which models a rectangular-shaped rigid solid. An actuated revolute joint object is used to define the rotation axis of the body. These objects have an additional flange connection. Thus, different elements of the Rotational Library can be attached in order to change the behaviour of the movement (PujanaArrese et al. 2006). Specifically, a damper has been connected in this case and a non-linear element known as bearingFriction which includes Coulomb friction owing to the axis joint and the arm. As has been described in the previous section, the possibility exists of coupling up to 8 plates of $1 \mathrm{~kg}$ each on one end of the arm. The model also features this option, for which purpose a bodyBox has been designed of the same size as the plates. Furthermore, the amount of weights may be configured using a global parameter.

The stops against which the arm impacts when it reaches the limits have also been modelled with bodyBox elements. The Talka class has been developed in order to model the impact between the arm and the stops mentioned before, of which two instances have been included in the model - one for each point of contact. The input for these objects is the relative distance between the point of impact of the arm and the mechanical stop. When the distance is detected as becoming negative the force of the impact is calculated based on a spring and damper model. The forces calculated are applied to the points of contact on the arm using force input elements known as frameForce.

Once all the mechanical components of the model have been checked, its pneumatic parts are now analyzed. The objects that make it possible for the pneumatic components to interact with the mechanical ones are connected to the anchorage points of each muscle. These objects (lineForce) describe a line of force between their two mechanical ports. The magnitude of this force depends on the actuator element connected to the ports. Obviously, in this case the actuators will be two objects of the class that models the pneumatic muscle.

The pneumatic interfaces of the muscles are connected to two of the out ports of the proportional valve. Two silencers have been fitted to the remaining two outputs that function as exhausts, as is the case of the real valve. Meanwhile, a source of pressure commanded externally 


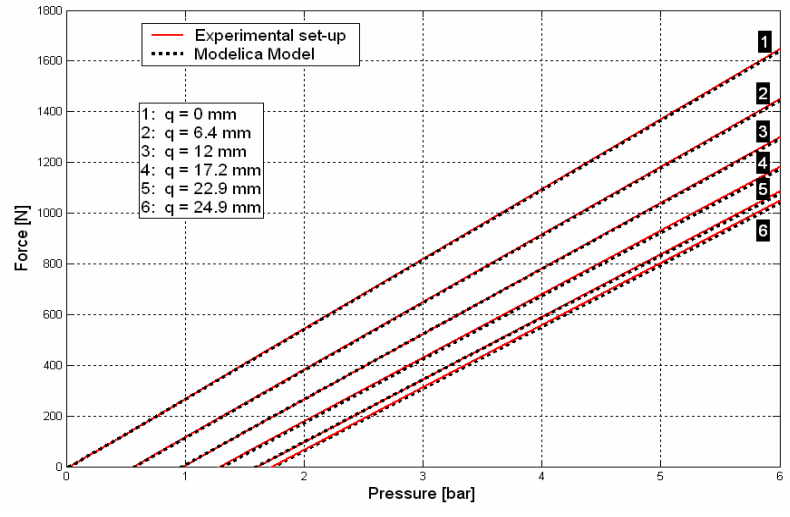

Figure 7: Results of model identification trials

is connected to the entry port. The valve model describes a proportional valve with second order spool dynamics in such a way that the spool position is almost proportional to the signal that commands it. When the valve is inactive, it attempts to close its five ports, letting a flow of leaks escape that is also modelled. To model the circuit pressure dynamics, this has been completed by connecting a lumpedVolume object to each port of the valve. All the elements mentioned in this paragraph belong to the Pneulib commercial library.

\section{EXPERIMENTAL VALIDATION OF MODELS}

In order to validate the equation (4) which determines the force exerted by the pneumatic muscle and to identify its coefficients, a series of experimental trials are carried out on the prototype constructed. Development of the experience involves keeping the muscle in a state of constant contraction and slowly increasing the pressure, recording the force exerted on a load cell. In this way, a family of straight lines is obtained with different contractions that relate force to pressure.

To carry out the trials, the muscle was firstly set at a determined, known length, thanks to the possibility of varying the lower stop of the prototype. Furthermore, the servovalve was removed so as to prevent undesired dynamic influences in such a way that pressurized air was introduced directly into the muscle. The experience involved blowing air into the muscle in order to make the arm descend; however, as the arm was positioned on the lower stop, it failed to move and therefore the length of the muscle remained constant. The air pressure was slowly varied in this position within the range of $0-6$ bar and the force exerted by the arm on the lower stop was recorded in the load cell. The real force exerted by the muscle was calculated using this data, together with geometric ratios.

The results obtained are shown in Figure 7. The different straight lines correspond to different constant contractions of the muscle. The upper straight line refers to the nominal length and the lower one to the maximum

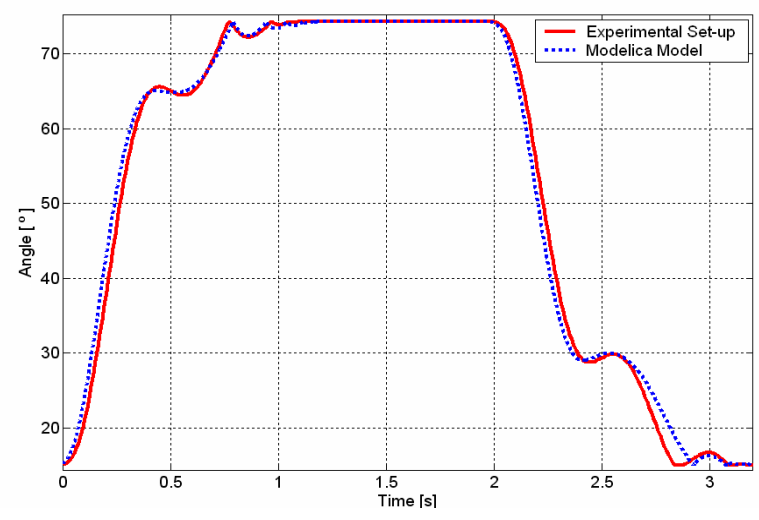

Figure 8: Results in open-loop being the load $3 \mathrm{~kg}$

contraction that may be experienced by the muscle in this model. It can be proven that the greater the length of the muscle, the greater the force it is capable of developing, as is gathered from the equation (4). It is also noted that the greater the contraction, the further the cut of the straight lines with the abscissa axis is from the origin - an effect which gave rise to the introduction of the correction term $\varphi(q)$ in the equation (4).

In the case described here and based on the experimental results shown in Figure 7 , the term $\varphi(q)$ has been identified by means of a $4^{\text {th }}$ order polynomial adjustment. Consequently, the values obtained for the different parameters of the model are:

$$
\begin{aligned}
& D_{1}=280 \quad D_{2}=-2.2 \quad D_{3}=0.033 \\
& \varphi(q)=0.00046 \cdot q^{4}-0.053 \cdot q^{3}+2.02 \cdot q^{2}-43.69 \cdot q+61.62
\end{aligned}
$$

The same Figure 7 shows the data obtained in simulation with the complete model of the prototype for the same experience. As is noted, the results in simulation coincide very well with the experimental data, which to a certain extent is normal, given that this experimental data forms the basis used for identifying the parameters of the muscle model.

To validate the complete model of the prototype made in Dymola/Modelica, open loop trials have initially been used on the model. The control element used is the servovalve, which accepts an input signal within the range of $0-10 \mathrm{~V}$. The prototype has been rotated in order to prevent the effects of gravity in such a way that the arm moves on a horizontal plane. A control signal has been applied to the servovalve: an impulse train of $\pm 0.9 \mathrm{~V}$ added to a central value of $+5 \mathrm{~V}$, over a period of 4 seconds. Trials have been carried out using different weights placed on the end of the arm. The arm completed its entire run from one end to the other using this open-loop signal, gently touching the stops. This trial has enabled the dynamics of the model to be checked and adjusted. An example of the results obtained may be viewed in Figure 8, together with those obtained in simulation, when there is a $3 \mathrm{~kg}$ weight on 
the end of the arm. The angle reflected in the ordinate axis of the figure corresponds to the angle measured from the vertical position (assuming the prototype has not been rotated).

As may be observed in the figure, both in the model and in the prototype there appears a vibration which originates from displacements in both directions in the intermediate area of movement, changing the dynamics of the system and making it slower. This vibration is due to the antagonistic structure of the muscles in the prototype, as the pressure and length vary inversely in each muscle while the arm is moving. This behaviour is also reflected in the results of the model, the key being the change in volume and the flattening at the ends during contraction in the model of the pneumatic muscle. Although the above-mentioned vibration is not evidenced in the results shown in many bibliographical references, it does appear in Tondu and Lopez (2000).

\section{CONCLUSIONS}

Pneumatic artificial muscles, or McKibben muscles, are very interesting for the development of robotic applications that feature low impedance in terms of their interaction with the environment, as may be the case with orthoses or certain wearable robots. In this paper, the modelling of a pneumatic muscle is shown in Dymola/Modelica, being the basis to model mechatronic systems based on such muscles. The component obtained is applied to the modelling of an experimental 1 DoF set-up, constructed using pneumatic muscles. The results of the experimental trials are used for an initial validation of the model.

\section{ACKNOWLEDGMENT}

The material used in this paper was partly supported by the Spanish Ministry of Education and Science and European FEDER Fund (research project DPI200614928-C02-01).

\section{REFERENCES}

Colbrunn, R.W.; G.M. Nelson; and R.D. Quinn. 2001. "Modeling of Braided Pneumatic Actuators for Robotic Control”. International Conference on Intelligent Robots and Systems (IROS'01), Maui, HI.

Petrovic, P.B. 2002. "Modeling and Control of an Artificial Muscle”. X Conference on Mechanical Vibrations. Timosoara, Belgrade.

Pujana-Arrese, A.; A. Martinez-Esnaola; F. Martínez; C.F. Nicolás; and J. Landaluze. 2006. "Construction machinery dynamic models for Real-Time training simulators". Industrial Simulation Conference ISC'2006. 5-7 June, Palermo, Italy.

Schröder, J.; D. Erol; K. Kawamura; and R. Dillmann. 2003. "Dynamic Pneumatic Actuator Model for a Model-Based Torque Controller”. IEEE Int Symp. On Computational Intelligence in Robotics and Automation.
Tondu, B. and P. Lopez. 2000. "Modeling and Control of McKibben Artificial Muscle Robot Actuators". IEEE Control Systems Magazine, April, pp.15-38.

Umetani, Y.; Y. Yamada; T. Morizono; T. Yoshida; and S. Aoki. 1999. "Skil Mate, wearable exoskelton robot". 16th IAARC/IFAC/IEEE International Symposium on Automationand Robotics in Construction (ISARC'99). September 22-24, Madrid, Spain.

\section{AUTHOR BIOGRAPHIES}

ARON PUJANA-ARRESE, JAVIER ARENAS, IBAN RETOLAZA, ANA MARTINEZ-ESNAOLA and JOSEBA LANDALUZE are with the Mechanical Design and Control Engineering departments of Ikerlan, a technological research centre located in the Basque Country (Spain). Their current research interests include design, modelling and control of biomimetic mechatronic systems, virtual prototyping and linear and non-linear robust control techniques. E-mail addresses are: APujana@ikerlan.es, JArenas@ikerlan.es, IRetolaza@ikerlan.es, AIMartinez@ikerlan.es and JLandaluze@ikerlan.es. 